\title{
Optical Spectroscopy at High Spatial Resolution with Fast Electrons
}

Luiz H. G. Tizei ${ }^{1}$, Sophie Meuret ${ }^{1}$, Noemie Bonnet ${ }^{1}$, François Treussart ${ }^{2}$, Bruno Daudin ${ }^{3}$, Bruno Gayral ${ }^{3}$ Romain Brourellier ${ }^{1}$, Anna Tararan ${ }^{1}$, Alberto Zobelli ${ }^{1}$, Yung-Chang Lin ${ }^{4}$, Kazu Suenaga ${ }^{4}$, Luiz. F. Zagonel $^{5}$, Odile Stéphan ${ }^{1}$ and Mathieu Kociak ${ }^{1}$

1. Laboratoire de Physique des Solides, UMR 8502 CNRS and Université Paris-Sud, Orsay, France

2. Laboratoire Aimé Cotton, CNRS, Université Paris Sud and ENS Paris-Saclay, Orsay, France

${ }^{3 .}$ CEA, INAC-PHELIQS, « Nanophysique est semiconducteurs group », Grenoble, France

4. National Institute of Advanced Industrial Science and Technology (AIST), Tsukuba, Japan

5. Instituto de Fiısica "Gleb Wataghin” Universidade Estadual de Campinas - Unicamp, Campinas, Sao Paulo, Brazil

Optical spectroscopy is traditionally performed using photon excitation (with a laser beam, for example). Typical emission experiments allow one to gather information of the different energy levels available in the system by measuring the intensity of the emitted light as a function of wavelength/energy. In a confocal optical microscopy setup, the ultimate spatial resolution is limited by the size of the detection volume (of the order of half a micrometer).

In this contribution, we will discuss the benefits and difficulties of performing optical spectroscopy using fast electrons as the excitation source (either in emission, using cathodoluminescence, [2], or absorption, by measuring electron energy loss, [3]). Much higher spatial resolution (from tens of nanometers to below a nanometer) is the evident attraction. Indeed, advances in electron optics (aberration correctors, monochromators and spectrometers) and improvements in electron microscope instrumentation (stages with cryogenic temperatures, including light collection systems) have brought electron spectroscopies closer to the energy resolution and detection sensitivity of standard optical spectroscopies.

To start with, we will describe cathodoluminescence (light emission from a material excited by electrons) experiments aimed at understanding exciton physics in two different systems: GaN quantum disks in AlN nanowires and hBN flakes. In the first system, we will discuss how the internal electric field present in the material influences light emission through energy band bending due to quantum confined Stark effect [4], brought by the heterestructure internal electric field. In Figure 1, a wavelength shift of $2 \mathrm{~nm}(22 \mathrm{meV})$ occurs due to the shielding of the internal field in a $1.5 \mathrm{~nm}$ wide (6 monolayers) quantum disk. This energy resolution is a factor of $15 \times$ better than that available in traditional electron energy loss experiments, but of the order of what is possible today with modern monochromators, as described later. For hBN, the influence of crystal stacking order on exciton emission energy [5] will be analyzed. A defect-related emission observed in the energy band gap of $\mathrm{hBN}$ has been identified using cathodoluminescence as a new single photon source emitting in the near UV range [6,7].

For 2D materials, excitons may also be probed using electron energy loss (EEL) spectroscopy. We will quickly revisit how an EEL spectrum is a direct measurement of $\varepsilon_{2}$ (imaginary part of the dielectric function), using hBN [8] as an example, which will be contrasted to cathodoluminescence experiments. Furthermore, current improvements in EELS energy resolution brought by new monochromators (energy resolution in the 5-30 meV range) give access to experiments in the optical range. To demonstrate this new possibility, we will analyze how the exciton absorption changes across the 
interface of a $\mathrm{MoS}_{2} \backslash \mathrm{MoSe}_{2}$ monolayer [9], showing that high spatial resolution (of the order of tens of nanometers) is still possible.

Finally, we will discuss our perspectives for experiments with new generation electron monochromators.

References:

[1] M Kociak and O Stéphan, Chem. Soc. Rev. 43 (2014) p. 3865.

[2] LF Zagonel et al, Nano Lett. 11 (2011) p. 568.

[3] J Nelayah et al Nat. Phys. 3 (2007) p. 348.

[4] LF Zagonel et al, Phys. Rev. B 93 (2016) p. 205410.

[5] R Bourrellier et al ACS Photon. 1 (2014) p. 857.

[6] LHG Tizei and M Kociak Phys. Rev. Lett. 110 (2013) p. 153604.

[7] R Bourrellier et al Nano Lett. 16 (2016) p. 4317.

[8] Z Liu et al Small 12 (2016) p. 252.

[9] LHG Tizei et al Phys. Rev. Lett. 114 (2015) p. 107601.
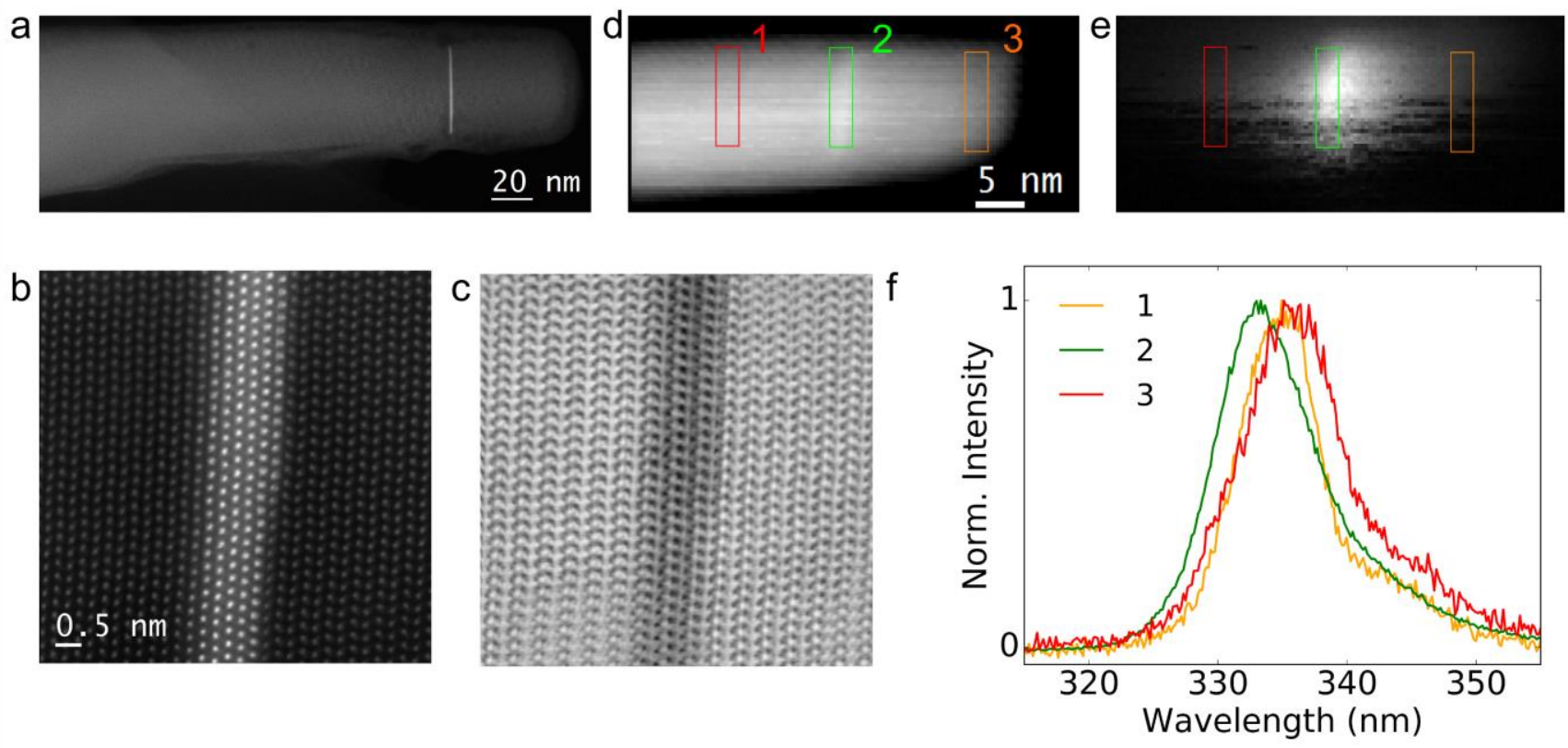

Figure 1. a-c) High angle annular dark field and annular bright field images of a $1.5 \mathrm{~nm}$ wide GaN quantum disk in a AlN nanowire. d) Annular dark field image of the same nanowire acquired at the same time as a spectrum image acquisition. e) Total light intensity emitted integrated around the emission peak. f) Normalized spectra averaged from the spectrum image in the regions marked 1,2 and 3. A wavelength shift of $2 \mathrm{~nm}(22 \mathrm{meV})$ is observed due to the quantum confined Stark effect. 